\title{
Bayesian Approach
}

National Cancer Institute

\section{Source}

National Cancer Institute. Bayesian Approach. NCI Thesaurus. Code C142403.

Approaches to data analysis that provide a posterior probability distribution for some parameter (e.g., treatment effect), derived from the observed data and a prior probability distribution for the parameter. The posterior distribution is then used as the basis for statistical inference. (ICH E9) 